\section{MS5-P7 Structural insights into malonyl-CoA reductase of 3-hydroxypropionate cycle}

Burak V. Kabasakal ${ }^{1}$, Burak V. Kabasakal ${ }^{1}$, Charles Cotton ${ }^{1}$, James W. Murray ${ }^{1}$

1. Department of Life Sciences, Wolfson Biochemistry Building, Imperial College London, Exhibition Road, London SW7 2AZ, U.K

email: burak.kabasakal11@imperial.ac.uk

The novel crystal structures of C-terminal part of Malonyl CoA reductase (MCR) from Chloroflexus aurantiacus and its NADPH bound form have been determined in $2.05 \breve{A}$ and $2.11 \breve{A}$, respectively. NADPH bound structure has a considerable conformational change compared to the native MCR. It forms an unusual hairpin structure near the NADPH binding site at the interface between the enzyme and solvent. The residues forming the hairpin structure are disordered at the native MCR. Furthermore, there is no significant difference between these two structures. NADPH binding site involves many consecutive hydrophobic residues which are necessary for nucleotide binding. Moreover, NADPH interacts with the polar residues which may play a crucial role in catalytic activity. These novel structures will elucidate the catalytic mechanism of MCR.

Keywords: protein crystallography, 3-HP cycle, carbon fixation, Malonyl CoA reductase

\section{MS5-P8 The first X-ray structure of} prokaryotic dipeptidyl peptidase III

Igor Sabljić ${ }^{1}$, Karl Gruber ${ }^{2}$, Peter Macheroux ${ }^{3}$, Marija Luić ${ }^{1}$

1. Division of Physical Chemistry, Ruđer Bošković Institute, Zagreb, Croatia

2. Institute of Molecular Biosciences, University of Graz, Graz, Austria

3. Institute of Biochemistry, Graz University of Technology, Graz, Austria

email: isabljic@irb.hr

Dipeptidyl peptidase III (DPP III) is a widely distributed cytosolic zinc peptidase from the M49 family, which hydrolyses dipeptides from the N-termini of its peptide substrates. Until now, most studies on DPP III were done on orthologs from eukaryotic organisms, showing that DPP III participates in the intracellular protein catabolism and oxidative stress response. The knowledge about prokaryotic DPP III is very scarce, particularly data on its catalytic mechanism, physiological function and structural characteristics are lacking. We have been studying two bacterial orthologs: DPP III from the human gut symbiont Bacteroides thetaiotaomicron $(B t)$ and from Caldithrix abyssi $(\mathrm{Ca})$, which inhabits hydrothermal vents. Both proteins were produced in Escherichia coli and then purified. Diverse crystallization screens were applied and wild-type $C a \mathrm{DPP}$ III crystals were obtained. Crystallization experiments with wild-type $B t$ DPP III failed probably due to charge heterogeneity. In order to overcome this problem, specific amino acid replacements (all cysteines were replaced by serines) were introduced and crystals of the variant protein were grown. Datasets for both DPP III proteins were collected at BESY II, Germany. Up to date, all efforts to solve the structures of the two proteins using molecular replacement method and anomalous dispersion of the zinc ions, were unsuccessful. Therefore, selenomethionine labeled BtDPP III, non-cysteine variant, was prepared and the crystals were obtained in the same crystallization condition as the non-labelled protein. Diffraction data up to $1.8 \AA$ resolution were collected at Elettra Trieste, Italy, and the first prokaryotic DPP III structure was solved using single-wavelength anomalous dispersion of the selenium atoms. BtDPP III crystallized in space group $P 3,21$ with unit cell dimensions $a=103.5 \AA$ and $c=141.6 \AA$ and one molecule per asymmetric unit. The overall fold is typical for a member of the M49 family with two domains separated by a wide cleft. The upper domain is mostly helical with a tetracoordinated zinc ion, while the lower domain contains mixed secondary structure elements with a five-stranded $\beta$-barrel core. The main structural difference between BtDPP III and eukaryotic DPP III is the absence of a 30 amino acid loop in upper domain. In human DPP III this loop contains the ETGE motif which is supposed to be responsible for binding KEAP1, a repressor protein from the Keap1-Nrf2 signaling pathway. 

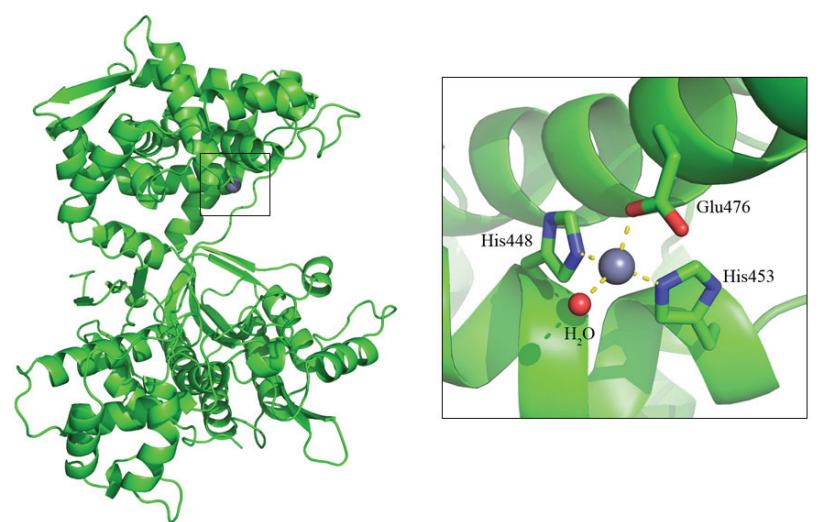

Figure 1. Overview of the BtDPP III structure. A two domains protein with characteristic M49 fold. The active site is located in the upper domain, containing a zinc ion coordinated by two histidines from the characteristic HECLGH motive, one glutamic acid from the EEARAD motive, and a water molecule.

Keywords: Dipeptidyl peptidase III, metallopeptidase, M49 family, Bacteroides thetaiotaomicron, Caldithrix abyssi

\section{MS5-P9 The cell-shape determining Csd6 protein from Helicobacter pylori constitutes a new family of L,D-carboxypeptidase \\ Hyoun Sook Kim ${ }^{1,2}$, Byung Woo Han ${ }^{1}$, Se Won Suh ${ }^{2}$}

1. Research Institute of Pharmaceutical Sciences, College of Pharmacy, Seoul National University, Seoul 151 742, Republic of Korea

2. Department of Chemistry, College of Natural Sciences, Seoul National University, Seoul 151 742, Republic of Korea

email: kobooks@gmail.com

Helicobacter pylori causes gastrointestinal diseases, including gastric cancer. Its high motility in the viscous gastric mucosa facilitates colonization of the human stomach, and depends on the helical cell shape and the flagella. In $H$. pylori, Csd6 is one of the cell shape-determining proteins that play key roles in determining the cell shape by alteration of cross-linking or by trimming of peptidoglycan muropeptides. Csd6 is also involved in deglycosylation of the flagellar protein FlaA. To better understand its function, we have carried out biochemical, biophysical, and structural characterizations. We show that Csd6 has three-domain architecture and exists as dimers in solution. The $\mathrm{N}$-terminal domain plays a key role in dimerization. The middle catalytic domain resembles those of L,D-transpeptidases and its pocket-shaped active-site is uniquely defined by the four loops I-IV, among which the loops I and III show the most distinct variations from the known L,D-transpeptidases. Our mass analyses confirm that Csd6 functions only as L,D-carboxypeptidase but not as L,D-transpeptidase. Our D-Ala-complexed structure reveals binding modes of both the substrate and product to the catalytic domain. The C-terminal nuclear transport factor 2-like domain possesses a deep pocket for possible binding of pseudaminic acid and our in silico docking supports its role in deglycosylation of flagellin. On the basis of our findings, we propose that $H$. pylori Csd6 and its homologs constitute a new family of L,D-carboxypeptidase. This work provides insights into the function of Csd6 in regulating the helical cell shape and motility of $H$. pylori.

Keywords: Csd6, cell shape, L,D-carboxypeptidase, Helicobacter pylori, HP0518, flagellin, peptidoglycan 